\title{
Relationship Between Late-Season Severity of Stewart's Bacterial Wilt and Seed Infection in Maize
}

C. C. Block, Plant Pathologist, USDA-ARS North Central Regional Plant Introduction Station, J. H. Hill, Professor, Department of Plant Pathology, and D. C. McGee, Professor, Department of Plant Pathology and Seed Science Center, Iowa State University, Ames 50011

\begin{abstract}
Block, C. C., Hill, J. H., and McGee, D. C. 1999. Relationship between late-season severity of Stewart's bacterial wilt and seed infection in maize. Plant Dis. 83:527-530.

The relationship between the amount of foliar disease on maize plants and seed contamination by the causal bacterium, Pantoea stewartii, was studied by comparing disease severity on adult plants with results from laboratory seed tests. Seventy-seven naturally infected maize lines (25 in 1990 and 52 in 1992) were selected and assigned to one of six disease severity classes based on the percentage of ear leaf tissue killed by Stewart's wilt: trace to $2 \%, 6$ to $14 \%, 25$ to $34 \%$, 35 to $49 \%, 50$ to $74 \%$, and 75 to $100 \%$. Ears were harvested from 10 to 20 plants representative of the disease class for each maize line, and seeds were tested by enzyme-linked immunosorbent assay (ELISA) of bulk-seed samples. Seed infection percentages were estimated from the bulk-test results by using statistical equations for group testing. The accuracy of the bulk-seed method for estimating seed infection was validated by comparison with 300-kernel single-seed tests. Infected seed was detected only from seed of plants with $\geq 25 \%$ disease severity; however, 45 of 63 such seed lots had no infection. Three seed lots had $>5 \%$ infected seeds, all from plants with $>50 \%$ disease severity. The results suggest a possible threshold level between 15 and $25 \%$ disease severity for Stewart's wilt on leaves before bacteria are detected in seed. This study describes a relatively simple method for estimating the incidence of infected seeds in a seed lot and contributes additional evidence to indicate that the chance of spreading $P$. stewartii from U.S.-produced maize seeds is low.
\end{abstract}

Additional keywords: Erwinia stewartii, seed pathology, Stewart's disease

Stewart's bacterial wilt is a disease of major phytosanitary importance because of concerns about the risk of seed transmission. Dozens of countries prohibit the importation of maize seed from the United States unless the seed is certified to be free of Pantoea stewartii (syn. Erwinia stewartii) (15), the causal agent of Stewart's wilt. $P$. stewartii is a nonmotile, gram-negative bacterium that infects maize leaves through feeding wounds made by Chaetocnema pulicaria Melsh. (7,16-18), the corn flea beetle. Bacteria multiply in and spread through the xylem $(8,11,18)$. Systemic infection occurs in susceptible plants when bacteria spread from the leaves to the vascular tissue of the stalk. Bacteria have been observed in the remnants of the vascular

Corresponding author: Charles C. Block

E-mail: ccblock@iastate.edu

Journal Paper J-17727 of the Iowa Agriculture and Home Economics Experiment Station, Ames, Iowa, Project 1018, supported by Hatch Act and State of Iowa funds.

Accepted for publication 1 March 1999

Publication no. D-1999-0412-01R

This article is in the public domain and not copyrightable. It may be freely reprinted with customary crediting of the source. The American Phytopathological Society, 1999. tissue at the base of the kernel, in the endosperm, and externally on the seed coat, but not in the embryo $(11,18,20)$.

Although seed infection has been clearly demonstrated, the evidence for seed transmission has been equivocal $(8,11,18,20,21)$. Recently, however, Block et al. (2) demonstrated seed transmission at a rate of $0.02 \%$ (rate adjusted to reflect only the infected kernel fraction) from seed of naturally infected plants and $0.14 \%$ from infected seed of artificially inoculated plants. Many of the seed lots used in that study were of generally poor quality (low germination), contained high percentages of infected kernels $(>35 \%)$, and would not have been exported commercially.

Commercial seed lots currently pass requirements for phytosanitary certification by field inspection of the seed production crop or by laboratory seed health testing. Field inspection is the major method for seed certification and is based on the assumption that incidences of infection on plants and seeds are related, although there are few diseases where this relationship has been validated (14). The presence of any level of Stewart's wilt in a field will cause that field to fail inspection. Seed lots that fail a Stewart's wilt field inspection can be certified if they pass certain laboratory tests, typically a seedling grow-out or a bulk-seed enzyme-linked immuno- sorbent assay (ELISA) (13). The grow-out test can be a good predictor of seed transmission in the field (14), but the Stewart's wilt ELISA is much more sensitive than the grow-out for identifying $P$. stewartiiinfected seed lots (D. McGee, personal communication). Experience in the Iowa State University Seed Health Testing Laboratory has shown that in most fields where the incidence or severity of Stewart's wilt was low, and the fields thus failed the field inspection, the pathogen was not detected by ELISA in the harvested seed (D. McGee, personal communication). Braun $(3,4)$ showed that resistant maize plants have the ability to limit lesion expansion by restricting bacterial multiplication and movement within the leaves. Thus, the relative severity of foliar disease on the seed-parent plants may indicate the likelihood of kernel infection.

The objectives of this study were to relate foliar disease severity on seed-parent plants to $P$. stewartii infection of harvested seed, as detected by ELISA, and to evaluate an easy, cost-effective bulk-sampling technique for estimating the percentage of infected kernels in a seed lot.

\section{MATERIALS AND METHODS}

Rating foliar disease severity. In 1990, Stewart's wilt severity was rated in singlerow plots of 23 open-pollinated (OP) plant introduction accessions and two hybrids grown at Crawfordsville, Iowa. Rows were $5 \mathrm{~m}$ long and $76 \mathrm{~cm}$ apart, with 15 to 20 plants per row. In 1992, similar ratings were made in 2-row plots of the hybrid B73 $\times$ Mo17, inbreds N28 and A632Ht, Jubilee sweet corn, and 48 OP accessions grown at Ames, Iowa. Rows were $8 \mathrm{~m}$ long and $90 \mathrm{~cm}$ apart, with 25 to 30 plants per row. Ratings were made between 1 September and 10 September of each year.

Stewart's wilt severity was assessed by visually rating the percentage of necrotic tissue on the ear leaf of 10 to 20 plants selected from each plot. Only plants with similar severity were selected to represent each maize line, and therefore each maize line could be assigned to a single disease class. Many of the accessions had 2 to 4 ears per plant, and for those plants an average disease rating was obtained by inspecting several leaves. The disease classes used were trace to $2 \%, 6$ to $14 \%, 25$ to $34 \%, 35$ to $49 \%, 50$ to $74 \%$, and 75 to 
$100 \%$ necrotic leaf tissue. No accessions were chosen to represent 3 to $5 \%$ or 15 to $24 \%$ disease classes because we preferred selecting maize lines that clearly fit into one class or another at the lower percentages. Ears from each plot were hand-harvested from the rated plants and dried with circulating air at $27^{\circ} \mathrm{C}$. Shelled seed was bulked from all ears of each maize line and stored at $5^{\circ} \mathrm{C}$ and $45 \%$ relative humidity.

Bulk-seed assays. For seed lots harvested in 1990, 250-kernel samples were subdivided into 50-kernel subsamples and tested by ELISA with a monoclonal antibody-based kit (13) according to the manufacturer's instructions (Agdia, Inc., Elkhart, IN). The subsamples were comminuted in a Waring blender for $1 \mathrm{~min}$ in $60 \mathrm{ml}$ of $0.02 \mathrm{M}$ phosphate-buffered saline containing $0.05 \%$ Tween 20 (PBS-T) and soaked for $1 \mathrm{~h}$. For the assay, duplicate $100-\mu \mathrm{l}$ samples were placed into wells of ELISA plates, and the absorbance value for each well was measured. Samples from three healthy kernels were used as negative controls on each plate, and the average absorbance of the negative controls was subtracted from each absorbance value. After this correction, absorbance values above 0.17 were defined as positive. Two positive control wells were included per plate.

For the 1992 seed lots, the sample size per seed lot was 150 kernels, with 25-kernel subsamples because several seed lots had all positive subsamples when 50-kernel subsamples were used. The subsamples were ground dry for $60 \mathrm{~s}$ in a Stein laboratory mill. Dry grinding was more convenient than wet grinding and did not affect sensitivity of the assay (C. C. Block, unpublished). The powder was soaked in $30 \mathrm{ml}$ of PBS-T for $1 \mathrm{~h}$ and tested by ELISA as previously described. Seed lots from which all six 25-kernel subsamples tested positive for $P$. stewartii were retested with 10 subsamples of 10 kernels each.

Estimating incidence of seed infection from bulk-seed assays. The estimated incidence of $P$. stewartii-infected kernels in each seed lot was determined by using statistical methods for group testing (i.e., pooled samples) based on the binomial model $(5,6)$.

The proportion of infected seeds $(p)$ was calculated from

$$
p=1-(1-Q)^{1 / n}
$$

where $n$ is the number of seeds per sample and $Q$ is the proportion of positive samples. The value $Q$ was obtained by dividing the number of positive samples (i.e., groups containing at least one infected seed) by the total number of samples tested. At least one negative sample (group) was required to estimate $p$. Seed infection percentages were obtained by multiplying $p$ by 100 .

Discussions of the statistical basis for group testing can be found in the literature cited by Hepworth (9), and several applications in plant pathology were cited by Hughes and Gottwald (10). Assumptions were made that infected seeds were randomly distributed in each seed lot and that the ELISA was completely effective for detecting a single infected kernel in the 25and 50-kernel sample sizes used in this study.

Confidence limits for $Q$ were obtained from the tables of Rohlf and Sokal (19) for proportions. The upper and lower confidence limits for $p$ were then calculated by substituting the respective upper and lower table values for $Q$ into equation 1 . Confidence limits were converted to percentages.

Comparison of the bulk-seed method with single-seed assays. In September 1992, individual plants were selected and tagged from a 0.4-ha (1-acre) field plot of hybrid B73 $\times$ KS4 grown at Ames. Plants were chosen to represent six disease severity classes: trace to $2 \%, 3$ to $5 \%, 6$ to $14 \%, 25$ to $34 \%, 35$ to $49 \%$, and 50 to $74 \%$ disease severity. Eight to 15 plants were identified from each class, and ears were handled as previously described. The seed lots were used to compare the bulk-seed ELISA with single-seed assays for estimating the percentage of $P$. stewartiiinfected kernels in a seed lot.

For the bulk-seed assays, 500-kernel samples from each disease class were subdivided into 50-kernel subsamples, ground dry, and tested by ELISA as previously described. The estimated percentage of infected kernels and associated confidence intervals were calculated in the manner described earlier. For the single-seed assays, 300 kernels from the 3 to $5 \%, 6$ to $14 \%, 25$ to $34 \%, 35$ to $49 \%$, and 50 to $74 \%$ disease classes were crushed one by one in a flat-bottomed Plattner mortar (Fisher Scientific, Chicago, IL). The mortar and pestle were wiped with ethanol between kernels. The powdered meal from each kernel was placed in a $12 \times 75 \mathrm{~mm}$ glass test tube containing $2.0 \mathrm{ml}$ of PBS-T buffer. Tubes were placed for $1 \mathrm{~h}$ on a reciprocating laboratory shaker at $100 \mathrm{rpm}$, and a $100-\mu$ sample from each tube was dispensed in one well of an ELISA plate. Positive and negative controls were included on each plate as previously described. Confidence limits for the singleseed tests were obtained by interpolation from binomial tables (19).

\section{RESULTS}

Comparison of bulk-seed method with single-seed assays. No seed infection was detected by the bulk-seed method from the trace to $2 \%, 3$ to $5 \%$, or 6 to $14 \%$ disease classes of hybrid B73 $\times$ KS4 (Table 1). Similarly, no seed infection was detected from the 3 to $5 \%$ or 6 to $14 \%$ disease classes by single-seed assays. Single-seed assays were not done for the trace to $2 \%$ seed lot because no seed infection was observed from the 3 to $5 \%$ and 6 to $14 \%$ disease classes. The estimated incidences of infected seed from the bulk-seed tests of the 25 to $34 \%, 35$ to $49 \%$, and 50 to $74 \%$ disease classes were similar to those obtained by the single-seed tests, about $0.2 \%$, $1 \%$, and 2 to $4 \%$ (Table 1 ). The confidence intervals from the single-seed tests overlapped the confidence intervals from the bulk-seed tests (Table 1).

Relationship between foliar disease and seed infection. The percentages of infected seed from the 77 maize lines surveyed, as estimated by bulk-seed ELISA, were consistent with those observed from similar disease classes in the comparison of bulk-seed and single-seed assays. Seed infection was not observed among five maize lines in the trace to $2 \%$ disease class or among nine lines in the 6 to $14 \%$ disease

Table 1. Comparison of bulk-seed enzyme-linked immunosorbent assay (ELISA) with single-seed assays for estimating the incidence of Pantoea stewartii-infected seed in hybrid B73 × KS4 in 1992

\begin{tabular}{|c|c|c|c|c|c|c|c|}
\hline \multirow{3}{*}{$\begin{array}{l}\text { Disease severity } \\
\text { class }(\%)\end{array}$} & \multicolumn{4}{|c|}{ Bulk-seed ELISA (50-seed samples) } & \multicolumn{3}{|c|}{ Single-seed assays (300 seeds sampled) } \\
\hline & \multirow{2}{*}{$\begin{array}{l}\text { Samples (of 10) } \\
\text { contaminated }\end{array}$} & \multirow{2}{*}{$\begin{array}{l}\text { Estimated seed } \\
\text { infection }(\%)^{\mathrm{a}}\end{array}$} & \multicolumn{2}{|c|}{$95 \%$ confidence limits } & \multirow{2}{*}{$\begin{array}{l}\text { Estimated seed } \\
\text { infection }(\%)^{\mathbf{b}}\end{array}$} & \multicolumn{2}{|c|}{$95 \%$ confidence limits } \\
\hline & & & Lower & Upper & & Lower & Upper \\
\hline Trace to 2 & 0 & 0 & & & Not tested & & \\
\hline 3 to 5 & 0 & 0 & 0.00 & 0.69 & 0.00 & 0.00 & 1.25 \\
\hline 6 to 14 & 0 & 0 & 0.00 & 0.69 & 0.00 & 0.00 & 1.25 \\
\hline 25 to 34 & 1 & 0.21 & 0.01 & 1.18 & 0.33 & 0.10 & 1.80 \\
\hline 35 to 49 & 3 & 0.71 & 0.18 & 1.91 & 1.00 & 0.30 & 2.92 \\
\hline 50 to 74 & 6 & 1.82 & 0.69 & 3.72 & 4.00 & 2.26 & 6.79 \\
\hline
\end{tabular}

a $\%$ seed infection was obtained from $100 \times p$ where $p=1-(1-Q)^{1 / n}, Q=$ number of contaminated samples $/ 10$, and $n=50$ seed.

b $\%$ seed infection was obtained from number of positive seeds/300. 
class (Table 2). Although quantitative measures of lesion size were not taken, the seed-parent plants in the trace to $2 \%$ severity class had small, restricted Stewart's wilt lesions, typically less than 2 to $3 \mathrm{~cm}$ long. Seed-parent plants in the 6 to $14 \%$ class had leaf lesions that were several centimeters long and sometimes coalesced with adjacent lesions, but showed no evidence of systemic infection. Systemic infection was defined as leaf to leaf or leaf to ear movement of $P$. stewartii via the vascular system of the plant. Symptoms of systemic infection included long lesions extending to the base of the leaf blade, and sometimes included leaf or plant wilting, stunting, and early death. Long lesions causing extensive necrosis, and characteristic of systemic infection, were observed on maize lines placed in the 25 to $34 \%, 35$ to $49 \%, 50$ to $74 \%$, and 75 to $100 \%$ disease severity classes.

Despite the generally high levels of foliar disease ( $\geq 25 \%$ severity) in 63 of the 77 maize lines surveyed, the actual number of infected seed lots and the incidence of seed infection was relatively low. Only two of 31 seed lots in the 25 to $34 \%$ class had $P$. stewartii-infected seed, and the incidence of seed infection in those lots was less than $1 \%$. Four of 16 seed lots in the 35 to $49 \%$ class, four of seven seed lots in the 50 to $74 \%$ class, and eight of nine seed lots in the 75 to $100 \%$ disease class had $P$. stewartii-infected seed (Table 2). The incidence of $P$. stewartii-infected seed did not reach $5 \%$ for any seed lot until foliar disease severity was at least $50 \%$. The highest incidence of seed infection from seed-parent plants with $<50 \%$ necrotic leaf tissue was $2.1 \%$, from the open-pollinated cultivar Longfellow Flint (PI 217408; 35 to $49 \%$ leaf area killed). Three seed lots had more than 5\% infected kernels, namely, Golden Bantam sweet corn (PI 255977; 50 to $74 \%$ leaf area killed) with $6.7 \%$ infected kernels, dent corn inbred $\mathrm{A} 632 \mathrm{Ht}$ with $10 \%$ infected kernels (50 to $74 \%$ leaf area killed), and Mohawk Round Nose flour corn (PI 483087; 75 to $100 \%$ leaf area killed) with $11.4 \%$ infected kernels.

\section{DISCUSSION}

The results suggest a direct relationship between severity of foliar symptoms of Stewart's wilt on seed-parent plants and the incidence of seed infection by $P$. stewartii, and also demonstrate that the mere presence of Stewart's wilt is not a good indicator of the likelihood of seed infection. $P$. stewartii-infected seed was found only in seed from parent plants with $\geq 25 \%$ necrotic leaf tissue. The absence of P. stewartii from seed of the five resistant (trace to 2\%) and nine moderately resistant (6 to 14\%) maize lines suggests a possible threshold level between 15 and $25 \%$ disease severity for Stewart's wilt on leaves before bacteria are detected in seed. We tried to select plants that were representa- tive of a single disease class for each maize line, although one cannot say with certainty that there was no overlap, particularly near 35 and $50 \%$ disease severity. To ensure a clear separation between disease classes in the range where systemic infection was likely to occur, we did not include a 15 to $24 \%$ disease class.

Anderson and Buzzell (1) compared the incidence of seed infection from a resistant and a susceptible hybrid and reported results that are consistent with our findings. They detected no seed infection from a hybrid with a small amount of leaf disease and $4 \%$ infected kernels from a severely infected hybrid. These data further support the conclusion of Khan et al. (12), who suggested that seed infection probably does not occur in nonsystemically infected plants.

Judging whether or not a plant is systemically infected can be difficult; however, it is relatively easy to recognize resistant maize lines with little chance for seed infection if the plants are inspected near maturity. In geographic areas where Stewart's wilt is normally a late-season or post-tasseling disease, the chance for seed infection is reduced, even on susceptible cultivars, because the pathogen has a relatively short time to colonize the plant. The low incidence of seed infection from many of the highly susceptible maize lines surveyed in this study was probably related to the late onset of disease.

There is currently no allowable level of seed-parent plant infection in phytosanitary field inspections, for either the incidence or the severity of plant infection. Under the zero tolerance regulations for Stewart's wilt, seed lots harvested from fields with any incidence of Stewart's wilt on seedparent plants are rejected, unless the seed passes laboratory testing. Thus, estimating the incidence of infected seeds in a seed lot is unnecessary, because any positive (i.e., infected) seed lots identified by laboratory testing are rejected. Nevertheless, it may prove valuable for assessing the risk of seed transmission to have such an estimate.

In an earlier report (2), we noted that 28 of 29 instances of seed transmission came from seed lots containing $>35 \%$ infected kernels. The rate of seed transmission was significantly higher from those seed lots than from naturally infected seed lots $(0.14$ and $0.022 \%$, respectively), even after adjustment for differences in the incidence of infected kernels. We suggested that there were probably additional factors that made seed transmission from the high-incidence seed lots more likely. Those seed lots contained many partially developed or slightly shrunken kernels, some of which still germinated. We suspect that kernels with extensive bacterial invasion are more likely candidates for seed transmission than are superficially colonized kernels. That study dealt almost exclusively with high-incidence seed lots, and the procedures used to dry and handle the seed were more likely to promote the survival of $P$. stewartii than the procedures normally used by commercial seed companies. Hence, the study was conservative in its estimates of the rates of seed transmission of $P$. stewartii. No studies have examined sufficiently large numbers of seedlings from naturally infected lower-incidence seed lots (e.g., 1 to $5 \%$ infected seeds) to demonstrate whether there might be a threshold incidence of seed infection below which seed transmission was so unlikely as to be of little phytosanitary concern. Such a study would require examining hundreds of thousand to millions of plants, depending on the desired level of confidence, and detecting no cases of seed transmission. Khan et al. (12) did not observe seed transmission from more than 75,000 plants grown from seed harvested from systemically infected plants. The incidence of kernel infection in their seed lots was probably quite low, as they were unable to detect $P$. stewartii in several seed lots. Hence, the actual number of infected seeds that were planted was too few to demonstrate a significantly lower probability of seed transmission.

If a tolerance were allowed for a certain percentage of infected seeds in a seed lot, the bulk-sampling ELISA technique would provide a simple and rapid means of estimating the incidence. Most seed-health testing laboratories already use multiple

Table 2. Number of maize lines assigned to each of six disease severity classes as related to the incidence of Pantoea stewartii-infected kernels (combined data from 1990 and 1992)

\begin{tabular}{lrccccccc}
\hline $\begin{array}{l}\text { Disease severity } \\
\text { class (\%) }\end{array}$ & \multicolumn{5}{c}{ Seed infection category } & \multirow{2}{*}{$\begin{array}{c}\text { Maize } \\
\text { lines }\end{array}$} \\
\cline { 2 - 6 } & $\mathbf{0 \%}$ & $\mathbf{0 . 5}$ to 1\% & $\mathbf{1 . 1}$ to 2.5\% & $\mathbf{2 . 6}$ to 5\% & $\mathbf{6}$ to 10\% & $>\mathbf{1 0 \%}$ & \\
\hline Trace to 2 & 5 & 0 & 0 & 0 & 0 & 0 & 5 \\
6 to 14 & 9 & 0 & 0 & 0 & 0 & 0 & 9 \\
25 to 34 & 29 & 2 & 0 & 0 & 0 & 0 & 31 \\
35 to 49 & 12 & 2 & 2 & 0 & 0 & 0 & 16 \\
50 to 74 & 3 & 0 & 0 & 2 & 2 & 0 & 7 \\
75 to 100 & 1 & 4 & 1 & 2 & 0 & 1 & 9 \\
Total & 59 & 8 & 3 & 4 & 2 & 1 & 77 \\
\hline
\end{tabular}

${ }^{a}$ Incidence of seed infection from each line was calculated from bulk-seed test results of five 50-seed samples (1990) or six 25-seed samples (1992).

${ }^{b}$ Ten to 20 plants were selected from each of 77 maize lines based on the percentage of necrotic tissue on the ear leaf (just below the ear). All plants from each maize line were in the same disease class, and the seed was pooled for ELISA. 
samples or batch ELISA testing. Hence, the data needed to estimate the incidence of infected seeds in a seed lot is potentially available. The proportion of infected seeds, $p$, determined by equation 1 , depends only on the number of seeds per batch $(n)$ and the proportion of positive batches, not on the total number of batches tested. For example, if two of four batches test positive, $p$ will be exactly the same as if 10 of 20 batches test positive. However, measures of the reliability of the estimate, such as confidence intervals, improve as more batches are tested (6).

For the bulk-seed method to be effective, a single infected seed must produce a sufficiently strong ELISA signal to compensate for dilution among the noninfected seeds. Hence, the sample size is limited by the detection sensitivity of the ELISA. This is of practical importance in the lowincidence seed lots, where the number of infected kernels per sample is generally zero or one. Seed lots with a high incidence of infected kernels usually have many infected kernels per sample, all contributing to the ELISA signal.

The seed infection percentage estimates obtained by bulk-seed testing (Table 1) seemed to be slightly lower than the percentages obtained by single-seed testing, although the confidence intervals overlapped. The differences between the singleseed and bulk-seed estimates could be due to chance, because only 10 samples were tested per seed lot in the bulk-seed assays. For example, in the 25 to $34 \%$ seed lot, the percentage of infected seeds estimated by the bulk-seed method was $0.21 \%$, compared with $0.33 \%$ estimated by single-seed testing. However, if a single infected seed had occurred in any one of the nine noncontaminated samples, the bulk-seed estimate would have been slightly higher $(0.44 \%)$ than the single-seed estimate. Similarly, if one more contaminated sample were detected in the 35 to $49 \%$ seed lot, the estimated percentage of infected kernels would have been $1.02 \%$, essentially identical to the single-seed estimate. In either case, the incidence of infected kernels was very low and the bulk seed method provided a useful estimate with far less effort than required by single-seed testing. There was some evidence for a dilution effect in the 50 to $74 \%$ seed lot. The bulk-seed estimate would have better approximated the single-seed estimate of $4 \%$ if there had been eight or nine contaminated batches, instead of six, yielding estimates of 3.2 or $4.5 \%$ infected seeds, respectively. The single-seed assays may have identified some superficially colonized kernels that might have gone undetected when diluted among the noninfected kernels in the bulk assay. However, obtaining precise estimates of the incidence of infected seeds in a seed lot is probably not as important as knowing whether the incidence is low or high. The combination of bulk-seed assays and statistical estimation offers a means for readily obtaining such data.

This study has demonstrated that a zero tolerance for Stewart's wilt incidence on seed-parent plants in seed production fields is unnecessarily restrictive. Disease severity was important in relation to the incidence of seed infection, but disease incidence on seed-parent plants was not. The study contributes additional evidence to indicate that the chance of spreading $P$. stewartii from U.S.-produced maize seeds is low. Sixty-three of the maize lines surveyed were very susceptible (>25\% disease severity) and not representative of the generally good resistance found in U.S. cultivars. Yet 45 of those 63 maize lines had no seed infection, and only 3 of 18 infected lines had seed infection levels greater than 5\%. The usefulness of ELISAbased seed health testing could be further expanded to estimate the percentage of infected seeds in a seed lot by using statistical methods for group testing.

\section{LITERATURE CITED}

1. Anderson, T. R., and Buzzell, R. I. 1986. Distribution and severity of Stewart's bacterial wilt of dent corn in Ontario, 1985. Can. Plant Dis. Surv. 66:23-25.

2. Block, C. C., McGee, D. C., and Hill, J. H. 1998. Seed transmission of Pantoea stewartii in field and sweet corn. Plant Dis. 82:775-780.

3. Braun, E. J. 1982. Ultrastructural investigation of resistant and susceptible maize inbreds infected with Erwinia stewartii. Phytopathology 72:159-166.

4. Braun, E. J. 1990. Colonization of resistant and susceptible maize plants by Erwinia stewartii strains differing in exopolysaccharide production. Physiol. Mol. Plant Pathol. 36:363-379.
5. Chen, C. L., and Swallow, W. H. 1990. Using group testing to estimate a proportion, and to test the binomial model. Biometrics 46:10351046.

6. Chiang, C. L., and Reeves, W. C. 1962. Statistical estimation of virus infection rates in mosquito vector populations. Am. J. Hyg. 75:377-391.

7. Elliott, C. 1935. Dissemination of bacterial wilt of corn. Ia. St. College J. Sci. 9:461-480.

8. Frutchey, C. W. 1936. A study of Stewart's disease of sweet corn caused by Phytomonas stewarti. Mich. Agric. Exp. Stn. Tech. Bull. 152.

9. Hepworth, G. 1996. Exact confidence intervals for proportions estimated by group testing. Biometrics 52:1134-1146.

10. Hughes, G., and Gottwald, T. R. 1998. Survey methods for assessment of citrus tristeza virus incidence. Phytopathology 88:715-723.

11. Ivanoff, S. S. 1933. Stewart's wilt disease of corn, with emphasis on the life history of Phytomonas stewarti in relation to pathogenesis. J. Agric. Res. 47:749-770.

12. Khan, A., Ries, S. M., and Pataky, J. K. 1996. Transmission of Erwinia stewartii through seed of resistant and susceptible field and sweet corn. Plant Dis. 80:398-403.

13. Lamka, G. L., Hill, J. H., McGee, D. C., and Braun, E. J. 1991. Development of an immunosorbent assay for seedborne Erwinia stewartii in corn seeds. Phytopathology 81:839-846.

14. McGee, D. C. 1995. Epidemiological approach to disease management through seed technology. Annu. Rev. Phytopathol. 33:445466.

15. Mergaert, J., Verdonck, L., and Kersters, K. 1993. Transfer of Erwinia ananas (synonym, Erwinia uredovora) and Erwinia stewartii to the genus Pantoea emend. as Pantoea ananas (Serrano 1928) comb. nov. and Pantoea stewartii (Smith 1898) comb. nov., respectively, and description of Pantoea stewartii subsp. indologenes subsp. nov. Int. J. Syst. Bacteriol. 43:162-173.

16. Poos, F. W., and Elliott, C. 1936. Certain insect vectors of Aplanobacter stewarti. J. Agric. Res. 52:585-608

17. Rand, F. V., and Cash, L. C. 1924. Further evidence of insect dissemination of bacterial wilt of corn. Science 59:67-69.

18. Rand, F. V., and Cash, L. C. 1933. Bacterial wilt of corn. U.S. Dep. Agric. Tech. Bull. 362.

19. Rohlf, F. J., and Sokal, R. E. 1995. Statistical Tables. 3rd ed. W. A. Freeman and Co., New York.

20. Smith, E. F. 1914. Stewart's disease of sweet corn (maize). Pages 89-147 in: Bacteria in Relation to Plant Diseases. Vol. 3, Vascular Diseases. Carnegie Institution of Washington, Washington, DC.

21. Stewart, F. C. 1897. A bacterial disease of sweet corn. N.Y. Agric. Exp. Stn. Bull. 130:422-439. 